\title{
Stellar dust production and composition in the Magellanic Clouds
}

\author{
F. Kemper \\ Academia Sinica, Institute of Astronomy and Astrophysics, PO Box 23-141, Taipei 10617, Taiwan, R.O.C.
}

(Received November 7, 2011; Revised April 18, 2012; Accepted April 22, 2012; Online published March 12, 2013)

\begin{abstract}
The dust reservoir in the interstellar medium of a galaxy is constantly being replenished by dust formed in the stellar winds of evolved stars. Due to their vicinity, nearby irregular dwarf galaxies the Magellanic Clouds provide an opportunity to obtain a global picture of the dust production in galaxies. The Small and Large Magellanic Clouds have been mapped with the Spitzer Space Telescope from 3.6 to $160 \mu \mathrm{m}$, and these wavelengths are especially suitable to study thermal dust emission. In addition, a large number of individual evolved stars have been targeted for 5-40 $\mu \mathrm{m}$ spectroscopy, revealing the mineralogy of these sources. Here I present an overview on the work done on determining the total dust production rate in the Large and Small Magellanic Clouds, as well as a first attempt at revealing the global composition of the freshly produced stardust.
\end{abstract}

Key words: Stars: AGB post-AGB stars, stars: mass loss, circumstellar matter, dust, Magellanic Clouds.

\section{Introduction}

Dust is a common ingredient of the interstellar medium (ISM) of galaxies. In the imaging obtained by optical observatories, such as the Hubble Space Telescope, dust manifests itself as dark lanes or patches that are obscuring the light from the stars. As such, dust was often seen as a nuisance, but with the advent of infrared astronomy, it has become obvious that the absorbed starlight is in fact reemitted in the form of thermal emission from dust grains, thus altering the shape of galaxy spectral energy distributions (SEDs). The dust-to-gas ratio in the ISM of galaxies is usually $\lesssim 1 \%$ by mass for galaxies with Solar metallicities (e.g. Draine et al., 2007), while typically about $30 \%$ of the energy emitted by such galaxies is in the form of thermal dust emission (e.g. Dale and Helou, 2002).

It is believed that dust in the interstellar medium is formed by generations of mass-losing evolved stars that gradually increase in metallicity with chemical galactic evolution (Morgan and Edmunds, 2003). Dust formation can occur in stars of all main-sequence masses, but the total dust budget is dominated by the more numerous Asymptotic Giant Branch (AGB) stars (with $M_{\mathrm{MS}}<8 M_{\odot}$ ), although Red Supergiants (RSGs; originating from $M_{\mathrm{MS}}>8 M_{\odot}$ ) are believed to be important contributors too, based on studies of stellar populations in the Solar neighbourhood (e.g., Whittet, 2003). AGB stars lose mass at a rate of $10^{-7}-10^{-6}$ $M_{\odot} \mathrm{yr}^{-1}$, and only for a short while, during the superwind phase, experience mass-loss rates of $10^{-4} M_{\odot} \mathrm{yr}^{-1}$ while RSGs can sustain mass loss at this high rate for longer (e.g. Habing and Olofsson, 2003; van Loon et al., 2005).

Copyright (C) The Society of Geomagnetism and Earth, Planetary and Space Sciences (SGEPSS); The Seismological Society of Japan; The Volcanological Society of Japan; The Geodetic Society of Japan; The Japanese Society for Planetary Sciences; TERRAPUB.

doi:10.5047/eps.2012.04.013

\section{The Magellanic Clouds}

Due to our vantage point within the Milky Way, and the extinction in the Galactic plane it is not possible to obtain a full global picture of the dust production from evolved stars in our own Galaxy. Instead, a global picture may be obtained from nearby external galaxies, and the Magellanic Clouds are obvious candidates for such a study. The Large and Small Magellanic Clouds are irregular dwarf galaxies at a distance of 50 and $61 \mathrm{kpc}$ respectively (Schaefer, 2008; Szewczyk et al., 2009), and both are seen almost face-on, resulting in a low optical depth along the line-of-sight, and very little confusion and few chance superpositions. Unfortunately, due to their vicinity to the much more massive Milky Way Galaxy, both Magellanic Clouds are tidally disrupted. Their relatively low metallicities of $Z_{\mathrm{LMC}} \sim 0.5 Z_{\odot}$ (Dufour et al., 1982) and $Z_{\mathrm{SMC}} \sim 0.2 Z_{\odot}$ (Bernard et al., 2008) make the Magellanic Clouds analogues for Milky Way type galaxies at cosmic times of $\sim 2$ and $\sim 0.7$ Gyr after the formation of our own Milky Way (Naab and Ostriker, 2006). The nearest spiral galaxy M31 (Andromeda) is at $750 \mathrm{kpc}$ significantly further away and to date, the Magellanic Clouds still provide the best compromise between distance and size to study the individual galaxy components, particularly stars.

\section{Dust Factories}

Thermal emission from dust, particularly warm $(\lesssim 1000 \mathrm{~K})$ newly formed circumstellar dust, is most prominent in the near- and mid-infrared. Thus, surveying a galaxy in the infrared, and identifying the point sources, will give us a handle on the dust production by evolved stars. Such surveys have been done in the past (e.g. DENIS (Cioni et al., 2000); MSX (Egan et al., 2001)), but using IRAC (Fazio et al., 2004) and MIPS (Rieke et al., 2004), the first modern infrared surveys of the LMC (Meixner et al., 2006) and SMC (Gordon et al., 2011) have been done, cataloguing over 8 million and 2 million infrared 
point sources in the LMC and SMC, respectively (Boyer et al., 2011). The SAGE surveys can be supplemented with near-infrared surveys, such as 2MASS (Skrutskie et al., 2006), the Vista Survey of Magellanic Clouds (VMC; Cioni et al., 2011), or the AKARI survey of the LMC (Ita et al., 2008).

\subsection{Source classification}

Blum et al. (2006) used the 2MASS data combined with the IRAC-[3.6] photometry to classify the dominant categories of dust producing evolved stars in the LMC. The first incarnation of the SAGE-LMC point source catalogue (Meixner et al., 2006) contained about 4 million sources, of which $\sim 820,000$ point sources have a $J$ and [3.6] magnitude measurement, mostly consisting of Red Giant Branch (RGB) stars $(\sim 650,000)$, which are not considered significant contributors of dust to the ISM. Only about $5 \%$ $(\sim 43,000)$ of the sources with a reliable $J$ and [3.6] magnitude are brighter than the tip of the RGB ([3.6] < 11.85) and thus potentially dust producing. These were subdivided using colour criteria, into carbon-rich AGB stars ( 7000), oxygen-rich AGB stars $(\sim 18,000)$, (Red) Supergiants $(\sim 1200)$ and extreme AGB stars $(\sim 1200)$. Other, non-dust-producing sources identified include foreground (Galactic) and background (extragalactic) objects.

Recently, Boyer et al. (2011) extended this method of classification to the SMC by including the SAGE-SMC survey (Gordon et al., 2011). They also revised the source counts for the LMC, using the most recent version of the SAGE-LMC point source catalogue, which now contains over 2 million sources with both $J$ and [3.6] band magnitudes, due to improved extraction and calibration methods ${ }^{1}$. Nevertheless, the number of point sources brighter than the tip of the RGB has been revised upward by only $\sim 7 \%$ to 45,870 , and the actual number of sources in each of the dust forming categories has changed at most by a similar percentage (see table 2 of Boyer et al., 2011).

The classifications performed by Blum et al. (2006) and Boyer et al. (2011), but also those done by other teams (e.g. VMC; Cioni et al., 2011) all work with sharply-defined colour and magnitude cuts to classify the point sources, while in reality the boundaries may not be that clear, and overlaps between the classes in colour-colour and colourmagnitude space may exist. Marengo and Sanchez (2009) have developed a more statistical approach to source classification using magnitudes and colours, based on a so-called Nearest Neighbour ( $k-\mathrm{NN})$ method. In this approach entries in a point source catalogue are assigned probabilities that they belong to a certain class of objects, based on their distance to, and the density (in colour-colour or colourmagnitude space) of a cloud of characteristic objects in that class. Thus, entries in the point source catalogue can have assigned probabilities to belonging to more than one object class, making this classification useful mostly in a statistical sense. The $k-\mathrm{NN}$ classification will be applied to all point sources in the SAGE-LMC and SAGE-SMC catalogues (Marengo et al., in prep.).

\footnotetext{
${ }^{1}$ Both the SAGE-LMC and SAGE-SMC point source catalogues are available through the NASA/IPAC Infrared Science Archive http://irsa.ipac.caltech.edu/applications/Gator/.
}

\subsection{Stellar dust production rates}

From these classifications, it is possible to estimate the dust production per source category in the LMC. Srinivasan et al. (2009) focused on the carbon-rich, oxygen-rich and extreme AGB star classifications from Blum et al. (2006) and using the infrared excess measured at IRAC 8.0 and MIPS $24 \mu \mathrm{m}$ found that the overall dust injection to the interstellar medium of the LMC is $2.74 \times 10^{-5} M_{\odot} \mathrm{yr}^{-1}$. The dust production is dominated by the category of extreme AGB stars, which are responsible for a dust production rate of $2.36 \times 10^{-5} M_{\odot} \mathrm{yr}^{-1}$, against 0.14 and $0.24 \times 10^{-5}$ $M_{\odot} \mathrm{yr}^{-1}$ for oxygen-rich and carbon-rich AGB stars respectively. An alternative estimate is presented by Matsuura et al. (2009) who used representative sources identified by their IRS (Houck et al., 2004) spectra to carve out a classification scheme in the [8.0] vs. [3.6]-[8.0] colour-magnitude diagram, distinguishing between carbon-rich and oxygenrich AGB stars. Using empirical mass-loss relations, they were able to estimate dust production rates of $4.3 \times 10^{-5}$ $M_{\odot} \mathrm{yr}^{-1}$ by carbon-rich AGB stars, and a lower limit of $4 \times 10^{-6} M_{\odot} \mathrm{yr}^{-1}$ for the oxygen-rich AGB stars. RSGs were also included in their analysis and responsible for at least $2 \times 10^{-6} M_{\odot} \mathrm{yr}^{-1}$ of dust production.

To estimate the dust injection in the SMC, the analysis performed by Srinivasan et al. (2009) for the LMC can be repeated. The colour cuts lead to the classification of almost 500,000 point sources in the SMC, with detections in $J$ and [3.6]. Of these, $\sim 19,000$ objects are brighter than the tip of the RGB. About 1700 stars are classified as carbon-rich AGB stars; about 4000 stars as oxygen-rich AGB stars, of which 227 as bright oxygen-rich AGB stars; a further 349 stars are extreme AGB stars; and around 3300 objects are thought to be RSGs. Thus, using the [8.0] and [24] $\mu \mathrm{m}$ excess, the overall dust production rate is thought to be an order of magnitude lower for the SMC, compared to the LMC (Boyer et al., 2012).

An important potential source of dust is ignored for both the LMC and the SMC: the dust production by supernova. This contribution is harder to estimate due to the incidental occurrence of supernovae, and the difficulties in establishing the dust production per supernova. Recently, Matsuura et al. (2011) provided a measurement of the dust mass associated with SN 1987A in the LMC, of 0.4-0.7 $M_{\odot}$, implying that supernovae can indeed be important dust producers, and providing an important step forward in establishing the dust contribution due to supernovae in the ISM of galaxies in the local universe.

\section{Mineralogy of Stardust}

The mineralogical make-up of the freshly formed stellar dust can be derived from infrared spectroscopy, as many common dust species have spectral resonances in the midinfrared, that can relatively easily be distinguished from each other.

Prior to Spitzer, a rare mineralogical analysis of dust produced by an LMC star was presented by Voors et al. (1999), who detected the presence of crystalline silicates in the luminous blue variable R71, observed with the Infrared Space Observatory (ISO; Kessler et al., 1996), using the Short Wavelength Spectrometer (SWS; de Graauw et al., 
1996). Trams et al. (1999) reported the presence of a silicate feature around a carbon-star in an observation with limited spectral range, but with a more extensive spectral range it was later shown that the 'emission feature' was really a lack of molecular absorption (Speck et al., 2006). Further ISO studies focused on the $10 \mu \mathrm{m}$ emission features in M-stars (Dijkstra et al., 2005), showing the presence of alumina and amorphous silicates, with the fraction of silicates increasing as the star evolves to higher mass loss rates.

With Spitzer it became possible to obtain 5-40 $\mu \mathrm{m} \mathrm{spec-}$ troscopy of many point sources in the LMC and SMC. Indeed, many observing programs were proposed, some targeting just a single or a few point sources, while other PIs observed a few hundred point sources with the IRS instrument. At several points during and after the mission, overviews of the spectral inventory of point sources in the Magellanic Clouds were given (Kastner et al., 2008; Sloan et al., 2008; Buchanan et al., 2009; Kemper et al., 2010). In total $\sim 1000$ point sources were observed in the LMC, and another $\sim 300$ in the SMC, covering a wide range of colours and magnitudes, and thus physical environments. The spectroscopic information can help to test the photometric classification schemes discussed in Subsection 3.1. The 297 staring mode observations performed in the context of the SAGE-Spec program (Kemper et al., 2010), were classified with the help of a decision tree (Woods et al., 2011). The result of this classification was complementary to and in agreement with the analysis done by Buchanan et al. (2009) and Kastner et al. (2008). A full spectral classification of the IRS staring mode observations in the LMC (Woods et $a l$, in prep.) and SMC (Ruffle et al., in prep.) is in preparation, the results of which will be used to test the $k$-NN photometric classification (Marengo et al., in prep.).

\subsection{Oxygen-rich stars}

Silicates dominate the spectra of both RSGs and oxygenrich AGB stars in the LMC. Dijkstra et al. (2005) studied a sample of $\mathrm{M}$ stars (of AGB and RSG origin) showing the $10 \mu \mathrm{m}$ silicate feature in emission or self-absorption in the ISO spectroscopy, and found that, while the spectra all show amorphous silicates, some of them also show the spectral signature of $\mathrm{Al}_{2} \mathrm{O}_{3}$ (alumina). The relative strength of the alumina signature to the spectrum decreases with increasing mass-loss rate, suggestive of a covering of the alumina grains by silicate mantles. At the highest mass-loss rates, the spectra are entirely dominated by silicate features.

When the IRS spectra of two typical AGB stars in the LMC, one of low and one of high mass-loss rate, are fitted with the same dust composition (Sargent et al., 2010), it is found that the best fit is produced by oxygen-deficient amorphous silicates (Ossenkopf et al., 1992), although other amorphous silicate profiles give good results too.

Some oxygen-rich stars show the sharp spectral resonances due to crystalline silicates. In addition to the presence of crystalline forsterite $\left(\mathrm{Mg}_{2} \mathrm{SiO}_{4}\right)$ around LBV R71 (Voors et al., 1999), Zijlstra et al. (2006) showed that oxygen-rich AGB star IRAS 05003-6712 also contains crystalline forsterite and enstatite $\left(\mathrm{MgSiO}_{3}\right)$ in its circumstellar shell. Low mass-loss rate LMC AGB star HV 2310 also shows crystalline silicates, particularly around 11.2 $\mu \mathrm{m}$, and the crystalline fraction of the silicates is around $7 \%$ by mass (Sloan et al., 2006a). A comprehensive study of all oxygen-rich AGB stars and RSG in the LMC and SMC for which IRS spectroscopy is available, shows that most objects do not show significant crystallinity in their silicates, although in some cases, the strength of the crystalline silicate features due to enstatite and forsterite at 23, 28 and $33 \mu \mathrm{m}$ can be up to $10 \%$ above the continuum level (Jones et al., in prep.). Given that at the wavelengths of the crystalline silicates resonances the intrinsic opacities of these crystalline silicates is much stronger than their amorphous counterparts, these measurements correspond to crystalline fractions of a few percent, at most. It needs to be emphasised however, that the lack of crystalline silicate features in the infrared spectrum does not mean that the crystalline silicates are absent; in particular in optically thin dust shells, they may simply be colder than the amorphous silicates (Kemper et al., 2001). Thus, I conclude for the purposes of this paper, that oxygen-rich AGB stars and RSGs in the LMC and SMC predominantly produce silicates, of an unspecified composition, but that a small fraction of these silicates may actually be due to crystalline enstatite and forsterite at the $\sim 5 \%$ level by mass.

\subsection{Carbon-rich stars}

The mineralogy of carbon-rich AGB stars in the Magellanic Clouds has been the subject of several studies. Silicon carbide ( $\mathrm{SiC}$ ), amorphous or graphitic carbon (C) and, in some cases, a solid-state feature at $30 \mu \mathrm{m}$, previously thought to be due to magnesium sulfide (MgS; Hony et al., 2002; Zhang et al., 2009), are seen in the IRS spectra of carbon-rich AGB stars in the LMC (Zijlstra et al., 2006) and the SMC (Sloan et al., 2006b). The $30 \mu \mathrm{m}$ feature is found to be present only in the redder, and hence colder, dust shells, where the dust temperature drops below $600 \mathrm{~K}$ (Zijlstra et al., 2006). In the low metallicity environments of the LMC and the SMC the mass balance between $\mathrm{SiC}$ and $\mathrm{C}$ in carbon stars will differ from those found in Galactic carbon stars (e.g. Sloan et al., 2006b), as the required carbon is produced by the star itself, but the silicon abundance reflects the composition of the natal cloud. Therefore, less silicon is available with lower metallicities. Indeed, Lagadec et al. (2007) confirmed that the SiC-feature-to-C-continuum ratio in the SMC is lower than in the LMC, but they also see an evolution with temperature ([6.4] - [9.3] colour) of the dust shells. While initially the $\mathrm{SiC}$ feature strength increases, with decreasing temperature, the $\mathrm{SiC}$ feature strength eventually drops. This is also seen by Leisenring et al. (2008), who study the strength of the $30 \mu \mathrm{m}$ emission feature in the same sequence, and extend the sample to bluer colours, indicative of lower mass-loss rates, particularly for the Milky Way comparison sample. While Lagadec et al. (2007) argue that $\mathrm{SiC}$ condenses first, followed by the condensation of $\mathrm{C}$ on top of the SiC, Leisenring et al. (2008) show that it is not possible with the LMC or SMC data set to establish the condensation sequence for $\mathrm{SiC}$ and $\mathrm{C}$. Both studies agree, however, that $\mathrm{MgS}$ condenses after SiC and $\mathrm{C}$ (Lagadec et al., 2007; Leisenring et al., 2008). Srinivasan et al. (2010) has determined the composition of the dust shell of a typical, albeit only one, C-rich AGB star, without the MgS emission feature at $30 \mu \mathrm{m}$, and finds that about $12 \%$ (in a range of $10-16 \%$ ) of the dust by mass is contained in $\mathrm{SiC}$, and that 
Table 1. Dust production in the LMC by various types of evolved stars, along with the adopted mineralogy in each source category. The bottom row summarises the total dust production rate and the mineralogy of the stellar dust production for the LMC. Dust production rates are taken from Matsuura et al. (2009) and Srinivasan et al. (2009). The typical compositions are discussed in Section 4 of the main text.

\begin{tabular}{|c|c|c|}
\hline Object type & $\begin{array}{l}\text { Mineralogy } \\
\text { (mass) }\end{array}$ & $\begin{array}{l}\text { Dust production rate } \\
\qquad\left(M_{\odot} \mathrm{yr}^{-1}\right)\end{array}$ \\
\hline O-rich AGB & $\begin{array}{l}95 \% \text { am. sil.; } \\
5 \% \text { cryst. sil. }\end{array}$ & $0.14->0.4 \times 10^{-5}$ \\
\hline C-rich AGB & $\begin{array}{c}88 \% \text { am. carbon.; } \\
12 \% \mathrm{SiC} \text {; var. \% } \mathrm{MgS}\end{array}$ & $0.24 \times 10^{-5}$ \\
\hline extreme AGB & $\begin{array}{c}88 \% \text { am. carbon; } \\
12 \% \mathrm{SiC} \text {; var. \% } \mathrm{MgS}\end{array}$ & $2.36-\lesssim 4.3 \times 10^{-5}$ \\
\hline RSG & $\begin{array}{l}95 \% \text { am. sil.; } \\
5 \% \text { cryst. sil. }\end{array}$ & $0.2 \times 10^{-5}$ \\
\hline Total & $\begin{array}{c}77 \% \text { am. carbon; } 11 \% \mathrm{SiC} \\
12 \% \text { am. sil.; < } 1 \% \text { cryst. sil.; var. } \mathrm{MgS}\end{array}$ & $(4 \pm 1) \times 10^{-5}$ \\
\hline
\end{tabular}

the remainder is in the form of amorphous carbon.

The class of extreme AGB stars is shown by Gruendl et al. (2008) to be made up of carbon-rich objects. In their sample of 13 objects, the $\mathrm{SiC}$ feature is actually seen in absorption, due to the high optical depth. Only two of the objects studied show the MgS silicate feature, casting doubt on how common this dust component really is in more evolved AGB stars. The mass fractions taken up by $\mathrm{SiC}, \mathrm{C}$ and possibly $\mathrm{MgS}$ in extreme AGB stars are not yet determined, but the subject of a future study (Speck et al., in prep.), and for the purpose of this work I will assume they are similar to what is derived for lower mass-loss rate C-rich AGB stars by Srinivasan et al. (2010).

\section{Results and Outlook}

The dust production rates and typical mineralogies for the dust factories in the LMC are summarised in Table 1. With the analysis so far, it is possible to derive the mineralogy of the stellar dust production input into the ISM for the LMC. I find that the majority $(\sim 77 \%)$ of the freshly produced stardust is in the form of amorphous or possibly graphitic carbon. Roughly equal amounts of stardust are taken up by $\mathrm{SiC}(\sim 11 \%)$ and amorphous silicates $(\sim 12 \%)$. Crystalline silicates, e.g. enstatite and forsterite, make up less than $1 \%$ of the dust production by evolved stars. An unspecified amount of MgS may also be produced by evolved stars. Apart from the fact that the $30 \mu \mathrm{m}$ feature is only seen in a subset of all C-rich AGB stars, and therefore hard to include in the analysis of a typical spectrum, it is also difficult to determine the dust mass contained in $\mathrm{MgS}$ in radiative transfer calculations, because of the lack of optical and near-infrared optical constants of $\mathrm{MgS}$ (Hony et al., 2002).

Another caveat is the absence of dust production by supernovae in Table 1. Although a reliable measurement of the dust mass associated with SN 1987A now exists (Matsuura et al., 2011), it is difficult to assess what fraction of that dust is produced by the supernova, and what fraction is simply processed or heated interstellar dust. In addition, we need to know how typical this supernova is in terms of dust production, and what the overall supernova rate is for the LMC and the SMC, to establish the dust production by supernovae. Finally, the mineralogy of supernova dust is not well known, with the mineralogy of supernova ejecta determined by interpreting single spectral features (Arendt et al., 1999; Rho et al., 2009).

Within the SAGE collaboration, we will continue to improve on the numbers presented in Table 1, as establishing the integrated composition of freshly produced stardust is indeed one of the goals of the SAGE-Spec project (Kemper et al., 2010). The typical compositions derived by Srinivasan et al. (2010) and Sargent et al. (2010) have been used to calculate a grid of SEDs, as a function of dust shell parameters (GRAMS; Srinivasan et al. (2011) and Sargent et al. (2011)). The SEDs in this grid will be fitted to the relevant photometric data in the SAGE-LMC catalogue, which will then allow us to update in particular the third column of Table 1, and calculate the total dust production accordingly.

In an alternative approach, the $k$-NN colour classification will allow for a statistical determination of the numbers in each source category (Marengo et al., in prep.). Along with a more detailed study of the mineralogy of a range of objects within source categories (e.g. Jones et al., in prep.; Speck et al., in prep.), the overall mineralogy will be better established as well. This procedure will also be carried out for the SMC.

Acknowledgments. This research has been supported by the National Science Council, under grant code NSC100-2112-M-001023-MY3.

\section{References}

Arendt, R. G., E. Dwek, and S. H. Moseley, Newly synthesized elements and pristine dust in the Cassiopeia A supernova remnant, Astrophys. J., 521, 234-245, 1999.

Bernard, J.-P. et al., Spitzer survey of the Large Magellanic Cloud, Surveying the Agents of a Galaxy's Evolution (SAGE). IV. Dust properties in the Interstellar Medium, Astron. J., 136, 919-945, 2008.

Blum, R. D. et al., Spitzer SAGE survey of the Large Magellanic Cloud. II. Evolved stars and infrared color magnitude diagrams, Astron. J., 132, 2034-2045, 2006.

Boyer, M. L. et al., Surveying the Agents of Galaxy Evolution in the tidally stripped, low metallicity Small Magellanic Cloud (SAGE-SMC). II. Cool evolved stars, Astron. J., 142, 103, 2011.

Boyer, M. L. et al., The dust budget of the Small Magellanic Cloud: Are asymptotic giant branch stars the primary dust source at low metallicity?, Astrophys. J., 748, 40, 2012.

Buchanan, C. L., J. H. Kastner, B. J. Hrivnak, and R. Sahai, Spitzer IRS spectra of luminous $8 \mu \mathrm{m}$ sources in the Large Magellanic Cloud: Testing color-based classifications, Astron. J., 138, 1597-1608, 2009. 
Cioni, M. R., R. P. van der Marel, C. Loup, and H. J. Habing, The tip of the red giant branch and distance of the Magellanic Clouds: results from the DENIS survey, Astron. Astrophys., 359, 601-614, 2000.

Cioni, M.-R. L. et al., The VMC survey. I. Strategy and first data, Astron. Astrophys., 527, A116, 2011.

Dale, D. A. and G. Helou, The infrared spectral energy distribution of normal star-forming galaxies: Calibration at far-infrared and submillimeter wavelengths, Astrophys. J., 576, 159-168, 2002.

de Graauw, T. et al., Observing with the ISO Short-Wavelength Spectrometer, Astron. Astrophys., 315, L49-L54, 1996.

Dijkstra, C., A. K. Speck, R. B. Reid, and P. Abraham, The $10 \mu \mathrm{m}$ feature of M-type stars in the Large Magellanic Cloud and the dust condensation sequence, Astrophys. J., 633, L133-L136, 2005.

Draine, B. T. et al., Dust masses, PAH abundances, and starlight intensities in the SINGS galaxy sample, Astrophys. J., 663, 866-894, 2007.

Dufour, R. J., G. A. Shields, and R. J. Talbot, Jr., The carbon abundance in the Magellanic Clouds from IUE observations of H II regions, Astrophys. J., 252, 461-473, 1982.

Egan, M. P., S. D. Van Dyk, and S. D. Price, MSX, 2MASS, and the Large Magellanic Cloud: A combined near- and mid-infrared view, Astron. J., 122, 1844-1860, 2001.

Fazio, G. G. et al., The Infrared Array Camera (IRAC) for the Spitzer Space Telescope, Astrophys. J. Suppl., 154, 10-17, 2004.

Gordon, K. D. et al., Surveying the Agents of Galaxy Evolution in the tidally stripped, low metallicity Small Magellanic Cloud (SAGE-SMC). I. Overview, Astron. J., 142, 102, 2011.

Gruendl, R. A., Y.-H. Chu, J. P. Seale, M. Matsuura, A. K. Speck, G. C. Sloan, and L. W. Looney, Discovery of extreme carbon stars in the Large Magellanic Cloud, Astrophys. J., 688, L9-L12, 2008.

Habing, H. J. and H. Olofsson, Asymptotic Giant Branch Stars, chap. 1 AGB stars: History, structure and characteristics, Springer, New York, 2003.

Hony, S., L. B. F. M. Waters, and A. G. G. M. Tielens, The carrier of the " 30 " $\mu \mathrm{m}$ emission feature in evolved stars. A simple model using magnesium sulfide, Astron. Astrophys., 390, 533-553, 2002.

Houck, J. R. et al., The Infrared Spectrograph (IRS) on the Spitzer Space Telescope, Astrophys. J. Suppl., 154, 18-24, 2004.

Ita, Y. et al., AKARI IRC survey of the Large Magellanic Cloud: Outline of the survey and initial results, Publ. Astron. Soc. Jpn., 60, 435-451, 2008.

Kastner, J. H., S. L. Thorndike, P. A. Romanczyk, C. L. Buchanan, B. J. Hrivnak, R. Sahai, and M. Egan, The Large Magellanic Cloud's top 250: Classification of the most luminous compact $8 \mu \mathrm{m}$ sources in the Large Magellanic Cloud, Astron. J., 136, 1221-1241, 2008.

Kemper, F., L. B. F. M. Waters, A. de Koter, and A. G. G. M. Tielens, Crystallinity versus mass-loss rate in asymptotic giant branch stars, Astron. Astrophys., 369, 132-141, 2001.

Kemper, F. et al., The SAGE-Spec Spitzer Legacy Program: The life cycle of dust and gas in the Large Magellanic Cloud, Publ. Astron. Soc. Pac., 122, 683-700, 2010.

Kessler, M. F. et al., The Infrared Space Observatory (ISO) mission, Astron. Astrophys., 315, L27-L31, 1996.

Lagadec, E. et al., Spitzer spectroscopy of carbon stars in the Small Magellanic Cloud, Mon. Not. R. Astron. Soc., 376, 1270-1284, 2007.

Leisenring, J. M., F. Kemper, and G. C. Sloan, Effects of metallicity on the chemical composition of carbon stars, Astrophys. J., 681, 1557-1573, 2008.

Marengo, M. and M. C. Sanchez, A $k-\mathrm{NN}$ method to classify rare astronomical sources: Photometric search of brown dwarfs with Spitzer/IRAC, Astron. J., 138, 63-75, 2009.

Matsuura, M. et al., The global gas and dust budget of the Large Magellanic Cloud: AGB stars and supernovae, and the impact on the ISM evolution, Mon. Not. R. Astron. Soc., 396, 918-934, 2009.

Matsuura, M. et al., Herschel detects a massive dust reservoir in Supernova 1987A, Science, 333, 1258-1261, 2011.

Meixner, M. et al., Spitzer survey of the Large Magellanic Cloud: Surveying the Agents of a Galaxy's Evolution (SAGE). I. Overview and initial results, Astron. J., 132, 2268-2288, 2006.

Morgan, H. L. and M. G. Edmunds, Dust formation in early galaxies, Mon. Not. R. Astron. Soc., 343, 427-442, 2003.

Naab, T. and J. P. Ostriker, A simple model for the evolution of disc galaxies: the Milky Way, Mon. Not. R. Astron. Soc., 366, 899-917, 2006.
Ossenkopf, V., T. Henning, and J. S. Mathis, Constraints on cosmic silicates, Astron. Astrophys., 261, 567-578, 1992.

Rho, J., W. T. Reach, A. Tappe, U. Hwang, J. D. Slavin, T. Kozasa, and L. Dunne, Spitzer observations of the young core-collapse supernova remnant 1E0102-72.3: Infrared ejecta emission and dust formation, Astrophys. J., 700, 579-596, 2009.

Rieke, G. H. et al., The Multiband Imaging Photometer for Spitzer (MIPS), Astrophys. J. Suppl., 154, 25-29, 2004.

Sargent, B. A. et al., The mass-loss return from evolved stars to the Large Magellanic Cloud. II. Dust properties for oxygen-rich Asymptotic Giant Branch stars, Astrophys. J., 716, 878-890, 2010.

Sargent, B. A., S. Srinivasan, and M. Meixner, The mass-loss return from evolved stars to the Large Magellanic Cloud. IV. Construction and validation of a grid of models for oxygen-rich AGB stars, Red Supergiants, and extreme AGB stars, Astrophys. J., 728, 93, 2011.

Schaefer, B. E., A problem with the clustering of recent measures of the distance to the Large Magellanic Cloud, Astron. J., 135, 112-119, 2008.

Skrutskie, M. F. et al., The Two Micron All Sky Survey (2MASS), Astron. J., 131, 1163-1183, 2006.

Sloan, G. C., D. Devost, J. Bernard-Salas, P. R. Wood, and J. R. Houck, The unusual silicate dust around HV 2310, an evolved star in the Large Magellanic Cloud, Astrophys. J., 638, 472-477, 2006 a.

Sloan, G. C., K. E. Kraemer, M. Matsuura, P. R. Wood, S. D. Price, and M. P. Egan, Mid-infrared spectroscopy of carbon stars in the Small Magellanic Cloud, Astrophys. J., 645, 1118-1130, 2006b.

Sloan, G. C., K. E. Kraemer, P. R. Wood, A. A. Zijlstra, J. Bernard-Salas, D. Devost, and J. R. Houck, The Magellanic zoo: Mid-infrared Spitzer spectroscopy of evolved stars and circumstellar dust in the Magellanic Clouds, Astrophys. J., 686, 1056-1081, 2008.

Speck, A. K., J. Cami, C. Markwick-Kemper, J. Leisenring, R. Szczerba, C. Dijkstra, S. Van Dyk, and M. Meixner, The unusual Spitzer spectrum of the carbon star IRAS 04496-6958: A different condensation sequence in the LMC?, Astrophys. J., 650, 892-900, 2006.

Srinivasan, S. et al., The mass loss return from evolved stars to the Large Magellanic Cloud: Empirical relations for excess emission at 8 and 24 $\mu \mathrm{m}$, Astron. J., 137, 4810-4823, 2009.

Srinivasan, S. et al., The mass-loss return from evolved stars to the Large Magellanic Cloud. III. Dust properties for carbon-rich asymptotic giant branch stars, Astron. Astrophys., 524, A49, 2010.

Srinivasan, S., B. A. Sargent, and M. Meixner, The mass-loss return from evolved stars to the Large Magellanic Cloud. V. The GRAMS carbonstar model grid, Astron. Astrophys., 532, A54, 2011.

Szewczyk, O., G. Pietrzyński, W. Gieren, A. Ciechanowska, F. Bresolin, and R. Kudritzki, The Araucaria Project: The distance to the Small Magellanic Cloud from near-infrared photometry of RR Lyrae variables, Astron. J., 138, 1661-1666, 2009.

Trams, N. R., J. T. van Loon, A. A. Zijlstra, C. Loup, M. A. T. Groenewegen, L. B. F. M. Waters, P. A. Whitelock, J. A. D. L. Blommaert, R. Siebenmorgen, and A. Heske, IRAS 04496-6958: A luminous carbon star with silicate dust in the Large Magellanic Cloud, Astron. Astrophys., 344, L17-L20, 1999.

van Loon, J. T., M.-R. L. Cioni, A. A. Zijlstra, and C. Loup, An empirical formula for the mass-loss rates of dust-enshrouded red supergiants and oxygen-rich Asymptotic Giant Branch stars, Astron. Astrophys., 438, 273-289, 2005.

Voors, R. H. M., L. B. F. M. Waters, P. W. Morris, N. R. Trams, A. de Koter, and J. Bouwman, Dust in R71: First detection of crystalline silicates in the LMC, Astron. Astrophys., 341, L67-L70, 1999.

Whittet, D. C. B., Dust in the Galactic Environment (second edition), Institute of Physics Publishing, London, 2003.

Woods, P. M. et al., The SAGE-Spec Spitzer Legacy programme: The life-cycle of dust and gas in the Large Magellanic Cloud-Point source classification I, Mon. Not. R. Astron. Soc., 411, 1597-1627, 2011.

Zhang, K., B. W. Jiang, and A. Li, On Magnesium Sulfide as the carrier of the $30 \mu \mathrm{m}$ emission feature in evolved stars, Astrophys. J., 702, 680685, 2009.

Zijlstra, A. A. et al., A Spitzer mid-infrared spectral survey of mass-losing carbon-stars in the Large Magellanic Cloud, Mon. Not. R. Astron. Soc., 370, 1961-1978, 2006.

F. Kemper (e-mail: ciska@asiaa.sinica.edu.tw) 